\title{
SMC based DTC-SVM control of five-phase permanent magnet synchronous motor drive
}

\author{
Fayçal Mehedi, Rachid Taleb, Abdelkadir Belhadj Djilali, Adil Yahdou \\ Laboratoire Génie Electrique et Energies Renouvelables (LGEER), \\ Electrical Engineering Department, Hassiba Benbouali University, Algeria
}

\begin{tabular}{l} 
Article Info \\
\hline Article history: \\
Received Feb 11, 2020 \\
Revised Apr 14, 2020 \\
Accepted Apr 16, 2020 \\
\hline Keywords: \\
Direct torque control \\
Five-phase permanent magnet \\
synchronous motor \\
Robustness \\
Sliding mode controller \\
Space vector modulation
\end{tabular}

\begin{abstract}
This article presents an improved Direct Torque Control (DTC) technique with space vector modulation (SVM) for a five-phase permanent magnet synchronous motor (PMSM) using a sliding mode speed control (SMC). The proposed control scheme of the five-phase PMSM combines the advantages of SMC control and the SVM algorithm. The SMC method insensitive to uncertainties, in particular external disturbances and parameter variations. In this paper, the SMC controller is used to control the rotor speed of the five-phase PMSM based on DTC-SVM. The rotor speed response, torque and stator flux are determined and compared with traditional control method. The simulations results confirm the validity and effectiveness of the proposed control technique in terms of performance and robustness against machine parameter variations (inertia variation). The efficiency of the proposed method applied on the five-phase PMSM is verified by the MATLAB/Simulink.
\end{abstract}

Copyright $@ 2020$ Institute of Advanced Engineering and Science. All rights reserved.

\section{Corresponding Author:}

Fayçal Mehedi,

Electrical Engineering Department,

Hassiba Benbouali University of Chlef, LGEER Laboratory,

BP. 78C, Ouled Farès 02180, Chlef, Algeria.

Email: fay.mehedi@gmail.com

\section{INTRODUCTION}

The Multiphase machines (MPMs) have more advantages compared to the 3 phase machines like stable speed response, low torque pulsations and high output power rating [1,2]. MPMs have gained attention in numerous fields of applications such as automobiles, ship propulsion and the wind energy conversion system [3, 4]. Direct torque control is the most important method used nowadays. The DTC strategy was first proposed for the induction motor (IM) in 1985 by Takahashi et al. [5]. The basic idea of this technique is a direct command of the stator flux and torque of IM without any pulse width modulation (PWM) strategy and proportional-integral (PI) controllers. However, this method is a simple structure compared to the field-oriented control (FOC) strategy and Scalar Control (V/f) [6, 7]. In this method, the switching table is used to control the inverter. On the other hand, the DTC method has many advantages, the fast dynamic response, lower parameter dependency, and reliability. But, the principal drawbacks, is the stator flux and torque ripples $[8,9]$. More recently, from the traditional DTC methods a new control technique called DTC with SVM has been developed. In [10], the DTC method was proposed based on SVM to command IM drive by using two PI controllers. In [11], the authors propose a variable speed control of PMSM using SVM-based DTC by employing using Ultra Sparse Matrix Converter (USMC). In this work the structures of DTC with switching table (DTC-ST) and the DTC with SVM of the five-phase PMSM have been presented. Traditional speed control structures which include PI regulator for application to an MPMs have some disadvantages such as parameter tuning complications, mediocre dynamic performances and reduced robustness $[12,13]$. In [14], the authors propose a four-level DTC strategy with the ANN controller 
by using neural PI controller of speed. In [15], a modified DTC-SVM with the enhancements of fuzzy logic control and closed loop stator flux estimation is presented. In this paper, we apply the sliding mode speed control (SMC) on the five-phase PMSM controlled by the DTC method using the SVM technique. This proposed control scheme is a simple method and minimizes the stator flux and torque ripple compared to conventional DTC. On the other hand, this proposed method is easy to implement and robust.

\section{MODEL OF FIVE-PHASE PMSM}

The five-phase PMSM stator voltage equation can be expressed in a rotating $d-q-x-y$ frame as follows $[16,17]$ :

$$
\left\{\begin{array}{l}
v_{d s}=R_{s} i_{d s}+\frac{d}{d t} \Phi_{d s}-w_{r} \Phi_{q s} \\
v_{q s}=R_{s} i_{q s}+\frac{d}{d t} \Phi_{q s}+w_{r} \Phi_{d s} \\
v_{x s}=R_{s} i_{x s}+\frac{d}{d t} \Phi_{x s} \\
v_{y s}=R_{s} i_{y s}+\frac{d}{d t} \Phi_{y s} \\
v_{0 s}=R_{s} i_{0 s}+\frac{d}{d t} \Phi_{s 0}
\end{array}\right.
$$

Where $v_{d s}, v_{q s}, v_{x \mathrm{~s}}$ and $v_{y s}$ are the stator voltages in the $d-q-x-y$ axis, $i_{d \mathrm{~s}}, i_{q \mathrm{~s}}, i_{x \mathrm{~s}}$ and $i_{y s}$ are the stator currents in $d-q-x-y, R_{s}$ is the stator resistance.

$$
\left\{\begin{array}{l}
\Phi_{d s}=L_{d} i_{d s}+\phi_{f} \\
\Phi_{q s}=L_{q} i_{q s} \\
\Phi_{x s}=L_{l s} i_{x s} \\
\Phi_{y s}=L_{l s} i_{y s} \\
\Phi_{s 0}=L_{l s} i_{0 s}
\end{array}\right.
$$

Where $L_{d}, L_{q}$ and $L_{l s}$ are inductances in the rotating frame.

The electromagnetic torque can be obtained as follows:

$$
T_{e}=\frac{5}{2} P\left(\left(L_{d}-L_{q}\right) i_{d s} i_{q s}+\phi_{f} i_{q s}\right)
$$

Mechanical motion equations are given by:

$$
J_{m} \frac{d w_{r}}{d t}=p T_{e}-p T_{r}-f_{m} w_{r}
$$

With $J_{m}$ is the inertia coefficient, $f_{m}$ is the viscous damping, $P$ is the number of poles pairs, and $T_{r}$ is the external load torque.

\section{DIRECT TORQUE CONTROL WITH SWITCHING TABLE}

In the conventional DTC, the stator flux amplitude $\phi_{s}{ }^{*}$ and the electromagnetic torque $T_{e}{ }^{*}$ are the reference signals which are compared with the estimated $\phi_{s}$ and $T_{e}$ values respectively in the stationary frame. The flux, torque errors and stator flux position are the inputs of switching Takahashi Table to deliver an appropriate voltage vector to the inverter. 
The stator flux linkages components in the stationary reference frame can be estimated by [16]:

$$
\left\{\begin{array}{l}
\Phi_{\alpha}=\int\left(v_{\alpha}-R_{s} i_{\alpha}\right) d t \\
\Phi_{\beta}=\int\left(v_{\beta}-R_{s} i_{\beta}\right) d t
\end{array}\right.
$$

The stator flux amplitude and phase are expressed using Concordia quantities, as follows:

$$
\left\{\begin{array}{l}
\Phi_{s}=\sqrt{\Phi_{\alpha}{ }^{2}+\Phi_{\beta}{ }^{2}} \\
\theta_{s}=\tan ^{-1} \frac{\Phi_{\beta}}{\Phi_{\alpha}}
\end{array}\right.
$$

The electromagnetic torque can be expressed in terms of stator current and flux as:

$$
T_{e}=\frac{5}{2} p\left(\Phi_{\alpha} i_{\beta}-\Phi_{\beta} i_{\alpha}\right)
$$

In DTC-ST control structure three control loops are applied: the control loop for motor angular speed with PI controller, the control loop for magnitude of the stator flux vector with hysteresis controller and the control loop for electromagnetic torque with hysteresis controller. The appropriate stator voltage vectors are chosen from the switching table which is presented in Table 1 . In this control system only the long voltage vectors have been used.

Table 1. Optimum active voltage vector look-up table

\begin{tabular}{cccccccccccc}
\hline & & $\mathrm{N}=1$ & $\mathrm{~N}=2$ & $\mathrm{~N}=3$ & $\mathrm{~N}=4$ & $\mathrm{~N}=5$ & $\mathrm{~N}=6$ & $\mathrm{~N}=7$ & $\mathrm{~N}=8$ & $\mathrm{~N}=9$ & $\mathrm{~N}=10$ \\
\hline \multirow{4}{*}{$d \phi=1$} & $d T=1$ & $V_{24}$ & $V_{28}$ & $V_{12}$ & $V_{14}$ & $V_{6}$ & $V_{7}$ & $V_{3}$ & $V_{19}$ & $V_{17}$ & $V_{25}$ \\
& $d T=0$ & $V_{0}$ & $V_{31}$ & $V_{0}$ & $V_{31}$ & $V_{0}$ & $V_{31}$ & $V_{0}$ & $V_{31}$ & $V_{0}$ & $V_{31}$ \\
& $d T=-1$ & $V_{17}$ & $V_{25}$ & $V_{24}$ & $V_{28}$ & $V_{12}$ & $V_{14}$ & $V_{6}$ & $V_{7}$ & $V_{3}$ & $V_{19}$ \\
& & & & & & & & & & & \\
& $d T=1$ & $V_{14}$ & $V_{6}$ & $V_{7}$ & $V_{3}$ & $V_{19}$ & $V_{17}$ & $V_{25}$ & $V_{24}$ & $V_{28}$ & $V_{12}$ \\
\multirow{4}{*}{$d \phi=0$} & $d T=0$ & $V_{31}$ & $V_{0}$ & $V_{31}$ & $V_{0}$ & $V_{31}$ & $V_{0}$ & $V_{31}$ & $V_{0}$ & $V_{31}$ & $V_{0}$ \\
& $d T=-1$ & $V_{7}$ & $V_{3}$ & $V_{19}$ & $V_{17}$ & $V_{25}$ & $V_{24}$ & $V_{28}$ & $V_{12}$ & $V_{14}$ & $V_{6}$ \\
\hline
\end{tabular}

The advantages of DTC method are as follows: structure independent on rotor parameters, no coordinate transformation, no current control loops. The disadvantages of classical DTC are: high switching losses provide by variable switching frequency, difficult implementation due to high sampling frequency $(25 \mu \mathrm{s})$.

\section{DIRECT TORQUE CONTROL WITH SPACE VECTOR MODULATION}

The traditional DTC algorithm is based on the instantaneous values and directly calculated the digital control signals for the inverter. DTC-SVM methods are based on averaged values, whereas the switching signals for the inverter are calculated by space vector modulator. This is the main difference between classical DTC and DTC-SVM control methods.

For the five-phase Voltage Source Inverter the total number of the state combinations of inverter switches is equal to $2^{5}=32$. In the set of all generated voltage vectors, thirty active vectors and two zero vectors can be identified [18]. The active switching vectors are divided in to three groups; small, medium and large switching vectors. Stator voltage space vectors generated by the five-phase VSI in the coordinate system $\alpha-\beta$ are presented in Figure 1 (a) and in the coordinate system $x-y$ are presented in Figure 1 (b).

It can be observed from Figure 1 that medium length space vectors of the $\alpha-\beta$ plane are mapped into medium length vectors in the $x-y$ plane and large vectors of the $\alpha-\beta$ plane are mapped into small vectors in the $x-y$ plane, and vice-versa [18].

In the applied SVM method the reference voltage vector lying in the considered sector is synthesized by using the appropriate switching times of two long and two medium voltage vectors, chosen from the same sector and of two zero voltage vectors. The case when the reference voltage vector $V_{s}^{*}$ is situated in sector 1 has been presented in Figure 2. In this case the reference voltage vector is synthesized with using: two long voltage vectors: $V_{25}, V_{24}$ two medium voltage vectors: $V_{16}, V_{29}$ and two zero voltage vectors: $V_{0}, V_{31}$. 


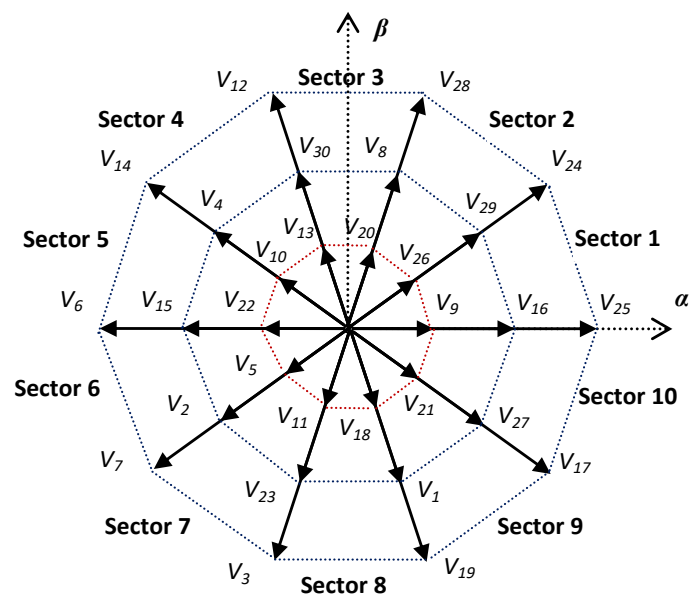

(a)

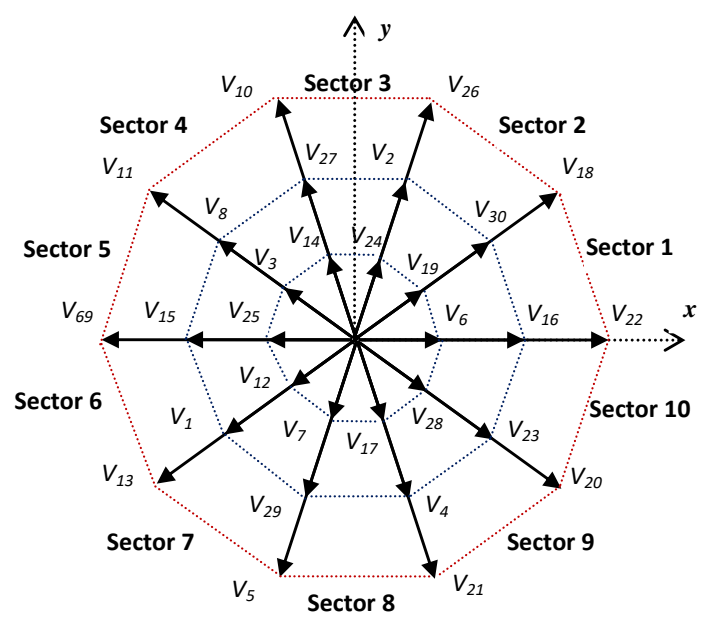

(b)

Figure 1. Voltage space vectors generated by the 5-phase VSI in coordinate systems: (a) $\alpha-\beta$ subspace, (b) $x-y$

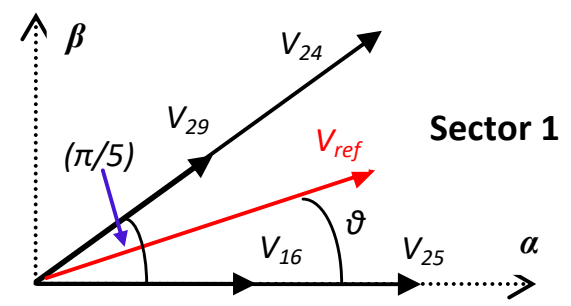

Figure 2. The principle of space vector modulation of 5-phase VSI

Switching times of individual voltage vectors are calculated as follows [18]:

$$
\left\{\begin{array}{l}
T_{a m}=\frac{\left|0.2764 V_{r e f}\right| \sin (k \pi / 5-\theta)}{\left|V_{m}\right| \sin (\pi / 5)} T_{s} \\
T_{a l}=\frac{\left|0.7236 V_{r e f}\right| \sin (k \pi / 5-\theta)}{\left|V_{l}\right| \sin (\pi / 5)} T_{s} \\
T_{b m}=\frac{\left|0.2764 V_{r e f}\right| \sin (\theta-(k-1) \pi / 5)}{\left|V_{m}\right| \sin (\pi / 5)} T_{s} \\
T_{b l}=\frac{\left|0.2764 V_{r e f}\right| \sin (\theta-(k-1) \pi / 5)}{\left|V_{l}\right| \sin (\pi / 5)} T_{s}
\end{array}\right.
$$

The dwell time equations used to describe the SVM algorithm in a sector $k$ can be written as:

$$
T_{0}=T_{s}-\left(T_{a m}+T_{a l}+T_{b m}+T_{b l}\right)
$$

where: $T_{a l}, T_{b l}$ switching times of long voltage vectors; $T_{a m}, T_{b m}$ switching times of medium voltage vectors; $T_{0}$ switching time of zero voltage vectors; $V_{\text {ref }}$ magnitude of the reference voltage vector; $T_{s}$ switching period; $\theta$ the angle of position of reference voltage vector; $k$ number of sector.

Figure 3 shows the block diagram of the DTC-SVM in a five-phase PMSM. 


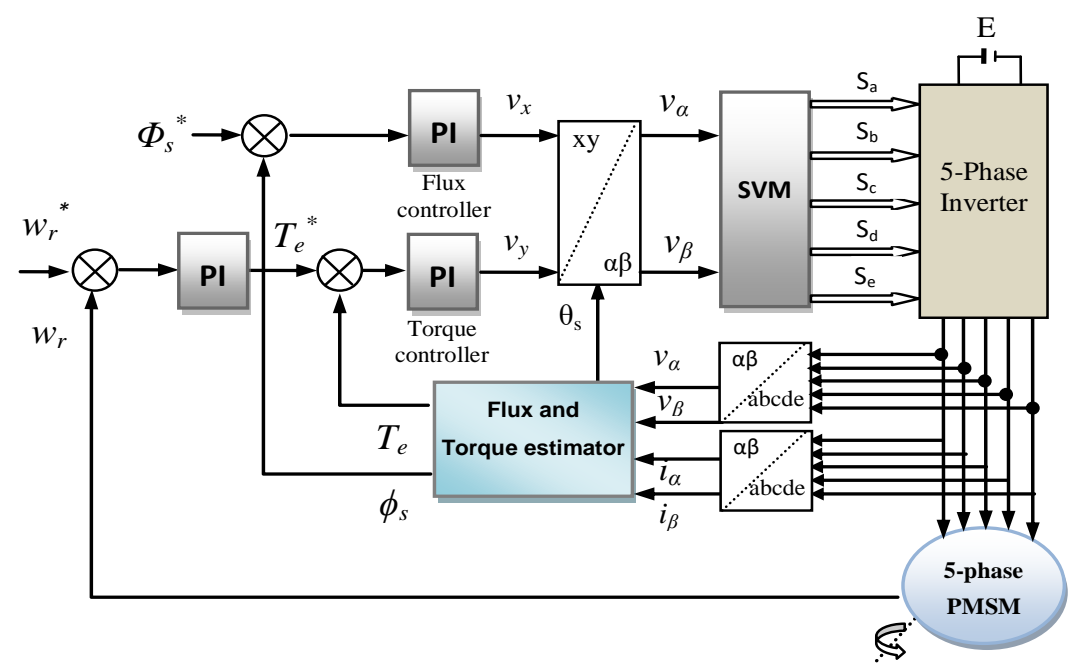

Figure 3. DTC-SVM control system of five-phase PMSM

In this scheme there are two proportional integral (PI) type controllers instead of hysteresis band regulate the torque and the magnitude of flux. The SVM unit produces the inverter control receives the reference voltages in a stator flux reference frame. The SVM principle is based on the switching between two adjacent active vectors and a zero vector during one switching period.

\section{THE SLIDING MODE SPEED CONTROL}

The approach of the SMC is based on the discontinuous function of state variables in the system that is used to create a "sliding surface". When this surface is reached, the discontinuous function guarded the trajectory on the surface of such so that the desired system dynamics is obtained [19, 20]. One considers the system described by the following state space equation:

$$
[\dot{X}]=A[X]+B[U]
$$

With $[X] \in R^{n}$, is the state vector; $[U] \in R^{m}$ is the control input vector; $[A]$ and $[B]$ are system parameter matrices. The first phase of the control design consists of choosing the number of the switching surfaces $S(x)$. Generally this number is equal the dimension of the control vector $[U]$. In order to ensure to convergence of the state variable $x$ to its reference value $x^{*},[21]$ proposes a general function of the switching surface:

$$
S(x)=\left(\frac{d}{d t}+\lambda\right)^{n-1} e(x)
$$

Here, $e(x)=x^{*}-x$ is the tracking error vector, $\lambda$ is a positive coefficient and $n$ is the relative degree.

The second phase consists to find the control law which meets the sufficiency conditions for the existence and reachability of a sliding mode such as

$$
S(x) \dot{S}(x)<0
$$

So that the state trajectory be attracted to the switching surface $S(x)=0$. A commonly used from of $U_{n}$ is a constant relay control.

$U_{n}=K_{x} \operatorname{sgn}(S(x))(13)$

$\operatorname{sgn}(\mathrm{S}(\mathrm{x}))$ is a sign function, which is defined as 


$$
\operatorname{sgn}(S(x))=\left\{\begin{aligned}
-1 & \text { if } S(x)<0 \\
1 & \text { if } S(x)>0
\end{aligned}\right.
$$

$k_{x}$ is a constant.

This introduces some undesirable chattering. Hence, one will substitute it by the function smooth [22-24].

$$
U_{n}=K_{x} \frac{S(x)}{|S(x)|+\xi_{x}}
$$

$\xi_{x}$ is small positive scalar.

The sliding surfaces are chosen according to (4) as follows:

$$
S\left(w_{r}\right)=w_{r}^{*}-w_{r}
$$

Based on the proposed switching surface, the speed control laws are:

$$
i_{q}^{*}=\frac{J_{m} \dot{w}_{r}{ }^{*}+p T_{r}+f_{m} w_{r}}{\frac{5}{2} p\left(\left(L_{d}-L_{q}\right) i_{d s}+\phi_{f}\right)}+\frac{S\left(w_{r}\right)}{\left|S\left(w_{r}\right)\right|+\xi_{w r}}
$$

The bloc diagram of the sliding mode speed control of the five-phase PMSM is given by Figure 4 .

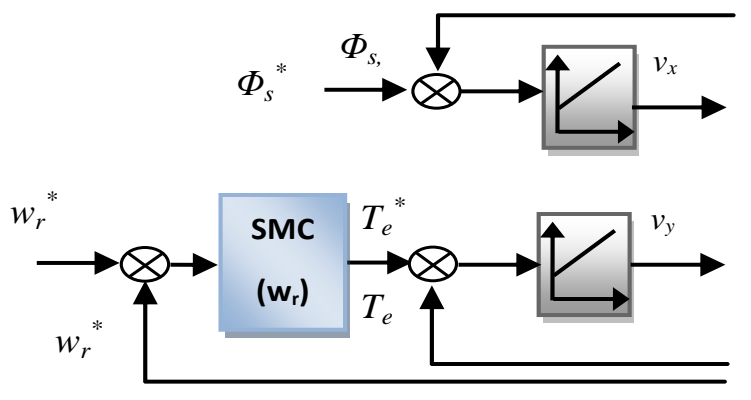

Figure 4. Block diagram of the sliding mode speed control

\section{SIMULATION RESULTS AND DISCUSSION}

In order to verify the effectiveness of the proposed scheme, simulations were carried out using Matlab/Simulink. The parameters of five-phase PMSM are as follows: $R_{s}=0.7 \Omega, P=2, \phi_{f}=0.5 \mathrm{web}, f_{m}=$ $0.005 \mathrm{Nm} / \mathrm{rad}_{\mathrm{s}} \mathrm{s}^{-1}, J_{m}=0.0025 \mathrm{Kg} / \mathrm{m}^{2}, L_{d}=0.0018 \mathrm{H}$ and $L_{q}=0.0042 \mathrm{H}[25]$.

In the objective to evaluate the performances of the control strategy, two categories of tests have been realized: sensitivity to the load torque variation and robustness (inertia variation).

Figure 5 show the responses of the five-phase PMSM under a sudden change in load torque from 0 $\mathrm{Nm}$ to $10 \mathrm{Nm}$ at $t=0.2 \mathrm{~s}$, after that the load torque is returned to $0 \mathrm{Nm}$ at $t=0.6 \mathrm{~s}$. In this test, the reference speed is step changed from $100 \mathrm{rad} / \mathrm{s}$ to $-100 \mathrm{rad} / \mathrm{s}$ at $t=0.8 \mathrm{~s}$. The speed response is shown in Figure 5 (a). The torque response for a step change of the load torque is shown in Figure 5 (b). The $\Phi_{\alpha \beta}$ curves in the DTC-ST and the DTC-SVM with SMC based speed controller are shown in Figure 5 (c). This figure express that the effect produced by the load torque variation is very clear on the speed curve of the system with PI controller, while the effects are almost negligible for the system with the SMC. It can be noticed that these last have a nearly perfect speed disturbance rejection (less than $2 \%$ ). While Figure 5 (b) it's clear that DTCSVM is able to reduced ripples level in torque compared with conventional DTC. In Figure 5 (c) the Lucas of stator flux is improved with very low ripple as compared with DTC-ST.

In order to test the robustness of the used controllers, the machine inertia has been doubled. The results presented in Figure 6 show that inertia variation presents a clear effect on the speed responses 
of all used PI controllers and that the effect appears more significant for DTC-ST than that with the DTCSVM with SMC based speed controller for the five-phase PMSM. Thus it can be concluded that these last are robust against this parameter variation.

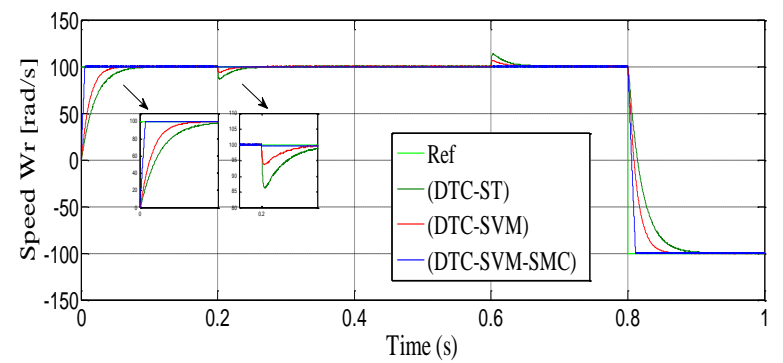

(a)

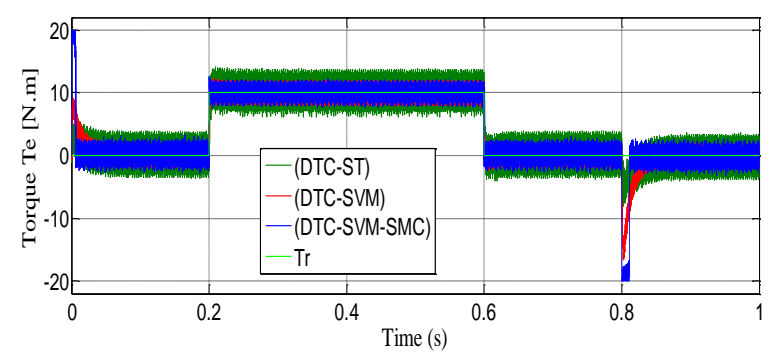

(b)
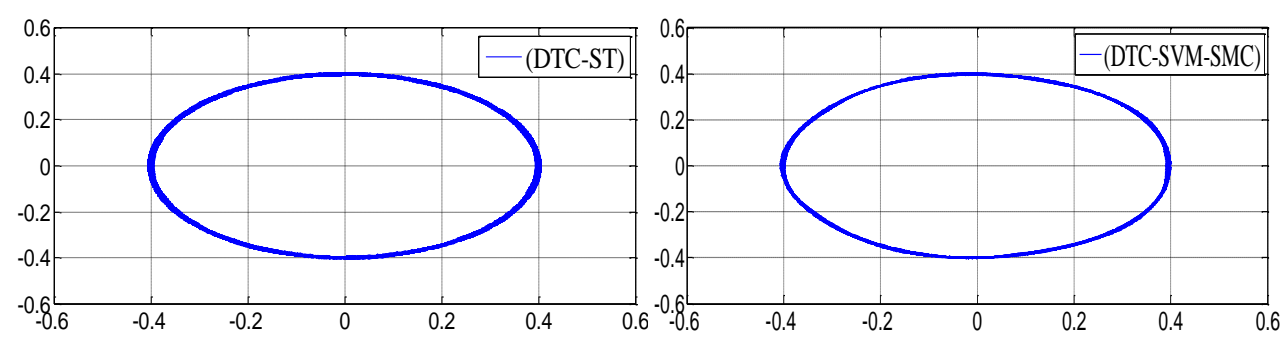

(c)

Figure 5. Dynamic responses of five-phase PMSM controlled by DTC-ST, DTC-SVM and DTC-SVM-SMC: (a) Rotor speed response, (b) Torque response, (c) Stator flux trajectory in $\alpha-\beta$ axis

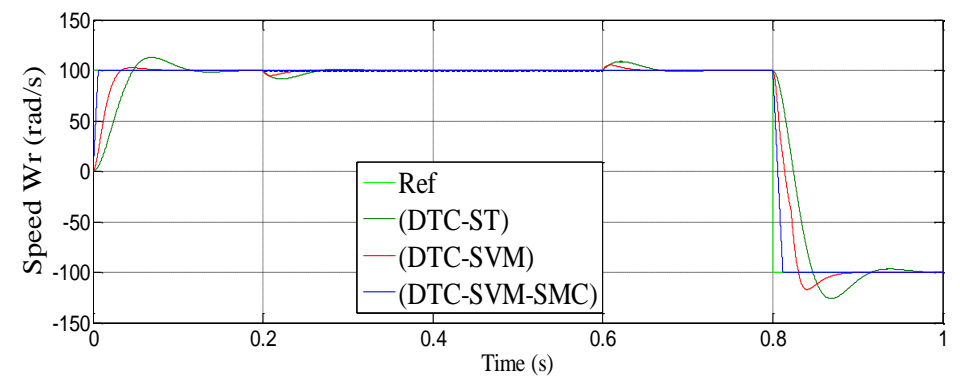

Figure 6. Robustness test $\left(J_{m}=2 * J_{m}\right)$

\section{CONCLUSION}

In this work, we presented the DTC technique based on the SVM algorithm for a five-phase PMSM using a sliding mode speed control. With results obtained from the simulation, it was clear that for the similar operation conditions, the proposed method presents high-quality performance compared to the traditional DTC method. The SMC can be considered as more robust under loads variations, minimizing the rise time and a very high robustness against machine parameter variations compared with the same results obtained for the traditional DTC system and DTC-SVM with PI based speed controller. For future works, it will be interesting to apply our proposed method in the default operating mode and set up a prototype in the lab to perform the experimental validation.

\section{REFERENCES}

[1] G. R. Catuogno, et al., "Fault tolerant control in six-phase PMSM under four open-circuits fault conditions," IEEE International Conference on Industrial Electronics, pp. 5754-5759, 2016. 
[2] L. Khaldi, et al., "Performance analysis of five-phase induction machine under unbalanced parameters," Journal Européen des Systèmes Automatisés (JESA), vol. 52, no. 5, pp. 521-526, 2019.

[3] M. Salehifar, et al., "Observer-based open transistor fault diagnosis and fault-tolerant control of five-phase permanent magnet motor drive for application in electric vehicles," IEEE IET Power Electronics, vol. 8, no. 1, pp. 76-87, 2015.

[4] E. Bounadja, et al., "A new DPC-SVM for matrix converter used in wind energy conversion system based on multiphase permanent magnet synchronous generator," Iranian Journal of Electrical and Electronic Engineering (IJEEE), vol. 15, no. 3, pp. 352-363, 2019.

[5] I. Takahashi, et al., "A new quick-response and high-effciency control strategy of an induction motor," IEEE Transaction induslly Applications, vol. IA-22, no. 5, pp. 820-827, 1986.

[6] H. Moghbeli, et al., "Transient and steady states analysis of traction motor drive with regenerative braking and using modified direct torque control (SVM-DTC)," The 6th International Power Electronics Drive Systems and Technologies Conference, pp. 615-620, 2015.

[7] M. Jannati, et al., "A new speed sensorless SVM-DTC in induction motor by using EKF," IEEE Student Conference on Research and Development, pp. 94-99, 2013.

[8] Z. Mekrini, et al.,"Fuzzy logic application for intelligent control of an asynchronous machine," Indonesian Journal of Electrical Engineering and Computer Science (IJEECS), vol. 7, no. 1, pp. 61-70, 2017.

[9] R. Logan, et al., "Improved torque control performance of direct torque control for 5-phase induction machine," International Journal of Power Electronics and Drive Systems (IJPEDS), vol. 3, no. 4, pp. 391-399, 2013.

[10] M. S. Raj, et al., "A modified direct torque control of induction motor using space vector modulation technique," Middle-East Journal of Scientific Research, vol. 20, no. 11, pp. 1572-1574, 2014.

[11] M. Yuhendri, et al., "Direct torque control strategy of PMSM employing ultra sparse matrix converter," International Journal of Power Electronics and Drive System (IJPEDS), vol. 9, no. 1, pp. 64-72, 2018.

[12] F. Mehedi, et al., "High-order sliding mode control of multiphase permanent magnet synchronous motor drive," The 2nd International Workshop on Signal Processing Applied to Rotating Machinery Diagnostics, SIGPROMD'2018, Djelfa, Algeria, 29-30, April 2018.

[13] F. Mehedi, et al., "Second order sliding mode control of two series-connected multi-phase permanent magnet synchronous motor," The Mediterranean Journal of Measurement and Control, vol. 13, no. 3, pp. 795-804, 2017.

[14] H. Benbouhenni, "Four-level direct torque control of permanent magnet synchronous motor based on neural networks with regulation speed using neural PI controller," Majlesi Journal of Mechatronic Systems, vol. 8, no. 4, pp. 965-975, 2019.

[15] J. D. Tan, et al., "Fuzzy logic enhanced direct torque control with space vector modulation," Indonesian Journal of Electrical Engineering and Computer Science (IJEECS), vol. 11, no. 2, pp. 704-710, 2018.

[16] K. Tounci, et al., "DTC-SVM of five-phase permanent magnet synchronous motor drive," 8th International Conference on Modelling, Identification and Control (ICMIC), pp. 103-108, 2016.

[17] Y. Zafari, et al., "Speed control of five-phase IPMSM through PI, SMC and FITSMC approaches under normal and open phase faulty conditions," Automatika Journal for Control, Measurement, Electronics, Computing and Communications, vol. 58, no. 4, pp. 506-519, 2017.

[18] U. Mahanta, et al., "Dynamic modeling and simulation of SVM-DTC of five phase induction motor," International Conference on Energy, Power and Environment (ICEPE), pp. 270-278, 2015.

[19] A. Yahdou, et al., "Sliding mode control of dual rotor wind turbine system," The Mediterranean Journal of Measurement and Control, vol. 11, no. 2, pp. 412-419, March 2015.

[20] S. Rafael, et al., "Sliding mode angular position control for an $8 / 6$ switched reluctance machine: Theoretical concept, design and experimental results," Electric Power Systems Research, vol. 129, pp. 62-74, 2015.

[21] J. J. Slotine, et al., "Applied nonlinear control," Englewood Cliffs, New Jersey: Prentice-Hall, 1991.

[22] H. Amimeur, et al., "Sliding mode control of a dual-stator induction generator for wind energy conversion systems," Electrical Power and Energy Systems, vol. 42, no. 1, pp. 60-70, 2012.

[23] Y. C. Koo, et al., "An enhanced distributed control-theoretic time synchronization protocol using sliding mode control for wireless sensor and actuator network," Indonesian Journal of Electrical Engineering and Computer Science (IJEECS), vol. 14, no. 2, pp. 688-696, 2019.

[24] D. Cherifi, et al., "Performance analysis of adaptive fuzzy sliding mode for nonlinear control of the doubly fed induction motor," Indonesian Journal of Electrical Engineering and Informatics, vol. 6, no. 4, pp. 436-447, 2018.

[25] Y. Borzou, et al., "Speed control of matrix converter-fed five-phase permanent magnet synchronous motors under unbalanced voltages," energies, vol. 10, no. 10, pp. 1-21, 2017.

\section{BIOGRAPHIES OF AUTHORS}

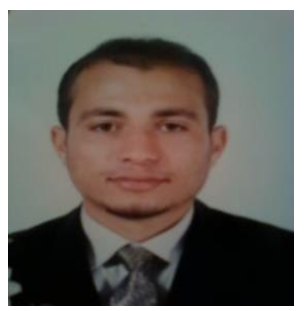

Fayçal Mehedi was born in 1984 in Chlef, Algeria. He is teacher in University of Chlef, Algeria. He received the Engineer degree in Electrical engineering and magister degree in Electrical engineering from University of Chlef, Algeria, in 2007 and 2011, respectively. He received a PhD in automatic from National Polytechnic School of Algiers, Algeria, in 2019. His research interests are in Electric Drive Control, Robust and Nonlinear Control, Observers, Multi-Machine System. 


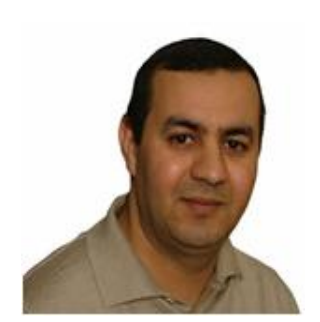

Rachid Taleb received the M.S. degree in electrical engineering from the Hassiba Benbouali University, Chlef, Algeria, in 2004 and the Ph.D. degree in electrical engineering from the Djillali Liabes University, Sidi Bel-Abbes, Algeria, in 2011. Currently he is a professor with the Department of Electrical Engineering, Hassiba Benbouali University. He is a team leader in the LGEER Laboratory (Laboratoire Génie Electrique et Energies Renouvelables). His research interest includes intelligent control, heuristic optimization, control theory of converters and converters for renewable energy sources.

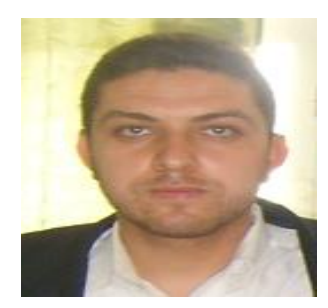

Abdelkadir Belhadj Djilali was born in Algeria, in 1982. He received the PhD degree in automatic from National Polytechnic School of Algiers, Algeria, in 2019. He is currently an associate professor at electrical engineering department at Hassiba Benbouali University. His research activities include power electronics and process control.

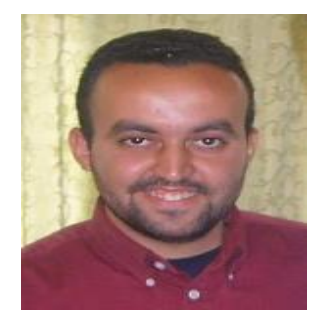

Adil Yahdou was born in Algeria in 1983. He is teacher in University of Chlef, Algeria. He received the $\mathrm{PhD}$ degree in automatic from National Polytechnic School of Algiers, Algeria, in 2017. He received a M.S. degree in Electrical Engineering from the University of Chlef, Algeria in 2011. His research activities include the study and application of robust control in the Dual rotor wind turbine system. 